\title{
Gestão zootécnica e genética informatizadas em pequenos ruminantes: uma revisão
}

\author{
[Computerized zootechnical and genetic management in small ruminants: a review]
}

\section{"Revisão/Review"}

\author{
Laylson Silva Borges ${ }^{1 *}$, Fernanda Samara Barbosa Rocha ${ }^{1}$, Vanessa Santos Neri ${ }^{1}$, \\ Flora Suzane Parente Maia ${ }^{1}$, Otávio Cury Costa Castro $^{2}$, José Elivalto Guimarães Campelo ${ }^{3}$, \\ José Lindenberg Rocha Sarmento ${ }^{3}$
}

\begin{abstract}
${ }^{1}$ Programa de Pós-Graduação em Ciência Animal, Universidade Federal do Piauí, Teresina-PI, Brasil. ${ }^{2}$ Programa de Pós-Graduação em Ciência da Computação, Universidade Federal do Piauí, Teresina-PI, Brasil. ${ }^{3}$ Departamento de Zootecnia, Universidade Federal do Piauí, Teresina-PI, Brasil.

*Autor para correspondência/Corresponding author: E-mail: laylson_borges@ hotmail.com
\end{abstract}

\begin{abstract}
Resumo
A criação de pequenos ruminantes tem se expandido em todos os estados brasileiros e, independentemente do objetivo da exploração, tem muito a contribuir para o desenvolvimento socioeconômico do país. Entretanto, esse setor enfrenta constantes desafios, como, por exemplo, a falta de informações e acesso às tecnologias, sendo a ausência de informatização uma das principais limitações para o registro de dados no campo. Dessa forma, objetivou-se, com esta revisão da literatura, compilar informações a respeito da utilização de ferramentas informatizadas aplicadas aos controles zootécnico e genético e à seleção de acasalamentos em pequenos ruminantes. Constatou-se que a adoção dessas ferramentas na ovinocultura e caprinocultura ainda é bastante limitada.
\end{abstract}

Palavras-chave: acasalamentos dirigidos; algoritmo genético; caprinocultura; informatização; ovinocultura.

\begin{abstract}
The production of small ruminants has increased in all Brazilian states, so that this activity has much to contribute to the socioeconomic development of the country, regardless of the farming purpose. However, this sector faces constant challenges, such as the lack of information and access to technologies, and the lack of computerization. The last one is one of the main factors hindering data recording in the field. Therefore, the aim of this review was to compile information about the use of computerized tools applied to zootechnical and genetic controls and mating strategies in small ruminants. It was found that the adoption of these tools in sheep and goat farming is still very limited.
\end{abstract}

Keywords: computerization; genetic algorithm; goat farming; mating strategies; sheep farming.

\section{Introdução}

O rápido crescimento da exploração de pequenos ruminantes está transformando o cenário dos sistemas produtivos no Brasil. De acordo com dados do IBGE (2016) existem aproximadamente 18,43 milhões de cabeças de ovinos e 9,78 milhões de caprinos no país. Deste total, 9,7 milhões de ovinos e 8 milhões de caprinos são criados na região Nordeste.

Apesar do grande efetivo e das potencialidades existentes, a região apresenta dificuldades para conhecer os índices produtivos e reprodutivos, principalmente em decorrência da falta de informações dos rebanhos e o uso de pouca tecnologia. Estes fatores contribuem para $\mathrm{o}$ desconhecimento de índices produtivos importantes e dificulta a adoção de tecnologias simples nos sistemas de criação, como seleção e direcionamento dos acasalamentos com base nas relações de parentesco (Carneiro et al., 2016).

Para os produtores que realizam a escrituração zootécnica de forma eficiente dos rebanhos, um dos desafios é a utilização dos dados 
gerados e a geração de informações que possam ser utilizadas nas tomadas de decisões. Uma alternativa é a adoção de ferramentas computacionais capazes de maximizar o uso de informações geradas a partir dos controles zootécnico e genético dos rebanhos. Dessa forma, o desenvolvimento de softwares voltados para este setor é de suma importância, pois estas atividades geram grande quantidade de variáveis que devem ser observadas e analisadas com maior exatidão.

Vale ressaltar que devido ao avanço da computação, é possível desenvolver sistemas de gerenciamento, controles zootécnico e genético para a ovino e caprinocultura, com a finalidade de facilitar a tomada de decisão do produtor e auxiliar no aumento em quantidade e qualidade do produto final. Além disso, o uso de recursos de inteligência computacional e de métodos eficientes para identificação de animais geneticamente superiores são de extrema importância para os sistemas de produção de ovinos e caprinos. No entanto, a adoção de inovações tecnológicas aplicada ao melhoramento genético animal depende do equilíbrio entre o que é possível, sob o ponto de vista tecnológico, e o que é aceitável, no contexto socioeconômico de um sistema de produção.

Dado o exposto, objetiva-se, com esta revisão da literatura, compilar informações a respeito da utilização de ferramentas informatizadas aplicadas aos controles zootécnico e genético e a seleção de acasalamentos em pequenos ruminantes.

\section{Panorama da ovinocultura e caprinocultura de corte no Brasil}

No Brasil, a oferta e a demanda de carne ovina e caprina está abaixo do potencial de produção, o que reflete a potencialidade da atividade para o setor no país, sobretudo na região Nordeste, onde esses pequenos ruminantes são caracterizados por apresentarem adaptação às condições edafoclimáticas e quase sempre estão ligados à agricultura familiar (Martins et al., 2015).

De acordo com Conrado et al. (2015) a criação de pequenos ruminantes, em especial os caprinos, é uma atividade de alta potencialidade, tanto social como econômica, para as populações de média e baixa renda do Nordeste brasileiro. Porém, nesta região predomina o sistema extensivo de criação, com baixa tecnificação e baixos resultados zootécnicos. Silvestre et al. (2015) relataram que muitos produtores vêm buscando melhores índices produtivos e reprodutivos nos seus rebanhos. Para isto utilizam meios de seleção dentro da raça ou recorrem à utilização de mais de uma raça através de cruzamentos, que é uma opção de alterar a produção e produtividade do plantel de forma mais rápida.

A ovino e a caprinocultura passam por um momento importante e decisivo em seu desenvolvimento. Assim, são indispensáveis à participação e o comprometimento de todos os atores envolvidos nesse setor de criação para o estabelecimento de estratégias e metas articuladas entre todos os elos da cadeia produtiva. Menezes Júnior et al. (2014) afirmaram que a criação de pequenos ruminantes representa um importante agente de inclusão e fonte de proteína para o semiárido nordestino.

Barros et al. (2009) destacaram a importância da criação de pequenos ruminantes no agronegócio brasileiro, configurando esta atividade como alternativa agropecuária apropriada para gerar crescimento econômico e benefícios reais em todas as regiões. Entretanto, eles chamam atenção para as incertezas que pairam sobre o mercado de produtos ovinos e caprinos, dentre os quais se destacam a clandestinidade dos abates destes animais, a falta de regularidade de oferta destes produtos, o hábito alimentar do brasileiro e o baixo poder aquisitivo da população. Este último, talvez se caracterize como principal entrave, tendo em vista o alto preço alcançado pela carne desses animais.

A criação de pequenos ruminantes é vista como uma fonte sustentável com excelentes possibilidades de rentabilidade econômica, o que a torna de suma importância para as regiões áridas e semiáridas. Entretanto, é de grande importância o desenvolvimento de novas tecnologias que sejam acessíveis e que facilitem a tomada de decisão por parte dos produtores, uma vez que os índices de produtividade não têm crescido satisfatoriamente, e em segunda instância a criação de novos produtos e de tecnologias privilegiam a qualidade do controle zootécnico e genético desses animais (Teixeira et al., 2013).

É relevante citar que em sistemas de criação que utilizam algum nível de tecnologia, torna-se evidente a melhoria na produção e reprodução, criando assim perspectivas de organização empresarial da atividade. Além disso, a criação de pequenos ruminantes de forma tecnificada, vem sendo considerada uma alternativa eficaz para o desenvolvimento da zona semiárida, pois aumenta a quantidade e qualidade do produto final, e 
consequentemente gera maior retorno financeiro para o produtor (França et al., 2016).

\section{Softwares aplicados aos controles zootécnico e genético e a seleção de acasalamentos em pequenos ruminantes}

O mapeamento de empresas ofertantes de softwares para o agronegócio em todo o território brasileiro foi realizado por Mendes et al. (2011). Eles verificaram que 162 empresas ofertavam 402 softwares desenvolvidos e/ou distribuídos em quatro categorias que abrangem as várias cadeias produtivas: administração/gerenciamento, manejo animal, cultivo vegetal e controle de processos e/ou de atividades rurais. Das 162 empresas pesquisadas, aproximadamente $58 \%$ possuem sede na região sudeste e $27,8 \%$ na região sul. Este fato torna-se ainda mais calamitoso ao se observar que estas empresas estão distribuídas em apenas 16 estados do país (Tabela 1).

Tabela 1. Distribuição das empresas privadas ofertantes de softwares para o agronegócio segundo região e unidade da federação da localização da sede.

\begin{tabular}{|c|c|c|c|c|c|}
\hline Região & Total de empresas & $\%$ & Estado & Total de empresas & $\%$ \\
\hline \multirow{4}{*}{ Sudeste } & \multirow{4}{*}{94} & \multirow{4}{*}{58,0} & São Paulo & 54 & 33,3 \\
\hline & & & Minas Gerais & 34 & 21,0 \\
\hline & & & Rio de Janeiro & 3 & 1,8 \\
\hline & & & Espírito Santo & 3 & 1,8 \\
\hline \multirow{3}{*}{ Sul } & \multirow{3}{*}{45} & \multirow{3}{*}{27,8} & Paraná & 23 & 14,2 \\
\hline & & & Rio Grande do Sul & 13 & 8,0 \\
\hline & & & Santa Catarina & 9 & 5,6 \\
\hline \multirow{4}{*}{$\begin{array}{c}\text { Centro- } \\
\text { Oeste }\end{array}$} & \multirow{4}{*}{15} & \multirow{4}{*}{9,3} & Mato Grosso & 7 & 4,3 \\
\hline & & & Goiás & 3 & 1,8 \\
\hline & & & Mato Grosso do Sul & 3 & 1,8 \\
\hline & & & Distrito Federal & 2 & 1,2 \\
\hline \multirow{4}{*}{ Nordeste } & \multirow{4}{*}{7} & \multirow{4}{*}{4,3} & Pernambuco & 4 & 2,5 \\
\hline & & & Sergipe & 1 & 0,6 \\
\hline & & & Bahia & 1 & 0,6 \\
\hline & & & Ceará & 1 & 0,6 \\
\hline Norte & 1 & 0,6 & Pará & 1 & 0,6 \\
\hline Total & 162 & 100 & & 162 & 100 \\
\hline
\end{tabular}

Fonte: Mendes et al. (2011)

É importante ressaltar que os softwares existentes para ovinos e caprinos no mercado nacional são, na maioria, adaptados de softwares desenvolvidos para bovinos não sendo específicos para controles zootécnico e genético de pequenos ruminantes. Sendo assim, é comum produtores adquirirem estes softwares pensando ter encontrado uma ferramenta que irá solucionar todos os problemas relacionados ao gerenciamento dos seus rebanhos. No entanto, depois de certo tempo de uso, observam que os mesmos não atendem as suas expectativas. Barbosa et al. (2000) afirmaram que isso pode ocorrer por vários motivos, dentre eles destacam-se: o software é muito complexo e não oferece um suporte satisfatório; não foi pedida nenhuma orientação; a empresa não ofereceu treinamento suficiente e não oferece segurança, além de atender somente parte das necessidades. Quando isso acontece, o sistema adquirido passa de ferramenta de solução para ferramenta de confusão, com isso o produtor poderá se tornar averso a qualquer tipo de investimento nesse sentido.

Dentre os softwares disponíveis no mercado destinados a ovino e a caprinocultura tem-se o Pecuária Brasil Ovinos, que oferece ao ovinocultor ferramentas que conferem facilidades no trabalho rotineiro, nas tomadas de decisão, na avaliação de desempenho zootécnico e no melhoramento genético do rebanho. Foi desenvolvido pela empresa Pecuária Brasil Assessoria, que tem como foco principal a gestão zootécnica e financeira na pecuária (Pecuária Brasil Assessoria, 2015).

O software Pecuária Brasil Ovinos disponibiliza como ferramenta de melhoramento genético animal o "Dossiê de matrizes", método que calcula índices informativos fenotípicos (taxa de prolificidade, idade ao primeiro parto, intervalo de partos e número de cobertura por prenhez) e de desempenhos (ganho de peso dos filhos) de cada ovelha do rebanho cadastrado. Assim, este software possibilita ao usuário verificar o 
desempenho individual de cada animal e o posicionamento de cada indivíduo no plantel a partir do fenótipo e não de valores genéticos. Isso facilita ao criador ou técnico identificar quais aspectos estão deficitários no rebanho, traçar as linhas de corte e identificar quais animais estão acima ou abaixo destas linhas.

Os animais que se encontram abaixo das linhas de corte serão classificados para descarte. Entretanto, essas linhas são definidas apenas a nível fenotípico, sem levar em consideração a base genética do animal, o que pode fazer com que um genótipo melhor seja observado como pior fenótipo e vice-versa, levando ao usuário do software a uma tomada de decisão errônea quanto a seleção e descarte dos animais.

Lôbo (2003) desenvolveu o programa GENECOC, que tem como objetivo principal estimular e assessorar produtores na escrituração zootécnica dos seus rebanhos, gerando informações de valores genéticos seguros e confiáveis que possam ser utilizadas na seleção dos melhores animais. Para isto, o programa utiliza um sistema de gerenciamento online por meio de um software em rede via internet, escrito em linguagens $P H P / H T M L / J a v a s c r i p t$ e acesso a um banco de dados PostgreSQL.

Para os rebanhos que já possuem estimativas de Diferença Esperada na Progênie (DEP), o GENECOC possui ferramentas de seleção de animais por mérito genético total, com a construção de um índice genético de seleção. Além disso, o programa estima a endogamia média do rebanho e fornece ao produtor uma listagem dos animais endogâmicos, como forma de auxiliar o produtor na orientação de acasalamentos.

Também está disponível no mercado o software CAPRIOVI, que foi desenvolvido pelo Grupo de Estudo em Genética e Melhoramento Animal (GEMA) em parceria com o Laboratório de Otimização de Software e Tecnologia (LOST) da Universidade Federal do Piauí. Para sua programação foram adotadas as linguagens Java e Fortran, com acesso a banco de dados MySQL, onde o usuário irá acessar e cadastrar os dados do rebanho a partir de computadores conectados à internet. O objetivo principal do CAPRIOVI é auxiliar o produtor nos controles zootécnico e genético, e na seleção de acasalamentos em caprinos e ovinos (Borges et al., 2017).

Por meio da utilização do software CAPRIOVI, o produtor é motivado a realizar a escrituração zootécnica do seu rebanho, permitindo um gerenciamento mais eficiente. A partir das informações cadastradas, serão gerados relatórios zootécnicos de produção, reprodução e genético, nos quais constam informações otimizadas, de forma a facilitar o entendimento e a tomada de decisão do ponto de vista de produção.

Com o uso do CAPRIOVI o produtor tem a sua disposição os métodos de seleção individual ou massal e índice de seleção para identificar animais geneticamente superiores para as características de interesse econômico. A partir da identificação dos melhores animais e considerando o interesse do produtor, este software pode ainda fazer a orientação de acasalamentos visando maximizar ganhos genéticos e minimizar a endogamia média dos rebanhos por meio de algoritmos de busca otimizada.

\section{Algoritmos de inteligência computacional aplicados à seleção de acasalamentos}

A seleção de acasalamentos consiste em realizar a melhor combinação de reprodutores e matrizes, visando à maximização do ganho genético e buscando também o controle da endogamia do rebanho (Carvalheiro et al., 2010; Jangarelli, 2014). Em programas de melhoramento genético, os acasalamentos seletivos podem ser utilizados para melhorar a estrutura genética da população para o próximo ciclo de seleção. Dessa forma, se bem planejados, esse procedimento pode promover o melhoramento genético dos rebanhos.

Santos et al. (2016) relataram que estratégias de acasalamento seletivo permitem o uso mais racional dos animais geneticamente superiores, de forma a alcançar os objetivos pré-estabelecidos em programas de melhoramento, além de possibilitar o uso de estratégias para a manutenção da variabilidade inter e intrarracial.

Atualmente, a otimização com algoritmos evolucionários está sendo usada para sanar problemas com a seleção de acasalamentos em bovinos e ovinos. Sendo assim, Carvalheiro et al. (2010) e Santos et al. (2016) mostraram a eficiência na utilização do algoritmo de evolução diferencial para a contribuição genética ótima, em termos de ganho genético com restrições para a coancestria. Os autores concluíram que a seleção de acasalamentos via algoritmo de evolução diferencial pode ser utilizada, pois oferecem diferentes cenários de ganho genético a partir de restrições no interesse nos componentes de função objetivo do algoritmo. 
A evolução diferencial é uma técnica estocástica de busca direta, fundamentada nos mecanismos de evolução natural das espécies. Esse algoritmo utiliza procedimentos de seleção de modo organizado com base no valor adaptativo dos indivíduos e operadores de mutação e recombinação (Storn e Price, 1997). Para Gomes et al. (2017) os métodos estocásticos procuram imitar processos ou fenômenos encontrados na natureza.

Os algoritmos evolucionários comparado a algoritmos como o Busca Tabu (Tabu Seach - TS), Colônia de Formigas (Ant Colony Optimization Algorithm - ACO) e o Recozimento Simulado (Simulated Annealing - SA) é a metodologia mais prática para resolver os problemas de otimização de forma eficiente. Vaisakh e Srinivas (2008) reiteraram que estes são empregados a problemas de otimização, pois geram procedimentos de buscas em vizinhanças que evitam a convergência prematura em ótimos locais proporcionando melhores soluções.

Em outra vertente, Carvalho et al. (2016) adotaram algoritmo genético para encontrar as melhores orientações de acasalamentos para um rebanho caprino da raça Anglo-nubiano, criados em sistema semi-intensivo. Eles inferiram que o algoritmo adotado indicou as melhores combinações de reprodutores e matrizes, obedecendo a relação estabelecida, visando a obtenção de progênies com maior ganho genético agregado e menor percentual de endogamia médio para o rebanho.

Destaca-se que os algoritmos genéticos (AGs) constituem uma técnica de busca e otimização altamente paralela, inspirada no princípio Darwiniano de seleção natural e reprodução genética (Monega et al., 2017). Santos et al. (2012) afirmaram que os AGs utilizam conceitos provenientes do princípio de seleção natural para abordar uma série ampla de problemas, em especial, de otimização. Apesar de aleatórios, os AGs não são 'caminhadas' aleatórias não direcionadas, pois exploram informações históricas para encontrar novos pontos de busca onde são esperados melhores desempenhos. Isto é feito através de processos iterativos, onde cada iteração pode ser considerada uma nova geração.

Kinghorn e Shepherd (1998) utilizando a teoria da contribuição genética, introduziram os algoritmos genéticos para otimização da seleção de acasalamentos. Para eles, a arquitetura necessária para sanar o problema com o uso de algoritmo genético envolve a representação do problema (as variáveis a serem otimizadas juntamente com todas as restrições existentes são representadas por incógnitas numéricas), a função objetivo (é a função das variáveis de decisão e define o problema de otimização como uma tarefa de minimização ou maximização) e o mecanismo de otimização (é a parte do programa que simula o processo de reprodução, mutação e seleção natural, em busca de soluções).

Carvalho et al. (2016) salientaram que os algoritmos genéticos buscam a solução para um problema de otimização, por meio de um conjunto de soluções candidatas, definida como população inicial, em seguida é atribuído um fitness (habilidade) a cada um dos indivíduos por meio de uma função de avaliação. Sendo assim, a cada geração, soluções relativamente "boas" se reproduzem, enquanto que soluções relativamente "ruins" são eliminadas. Para fazer a distinção entre diferentes soluções, é empregada a função de avaliação ou de adaptabilidade, que simula o papel de pressão exercida pelo ambiente sobre o indivíduo.

Nakamura et al. (2005) afirmaram que os AGs diferem dos métodos tradicionais de busca e otimização, principalmente por trabalharem com uma codificação do conjunto de parâmetros e não com os próprios parâmetros, bem como trabalham com toda população e não com um único ponto. Além disso, utilizam regras de transição probabilísticas e não impõem muitas das limitações encontradas nos métodos de busca tradicionais.

Além dos algoritmos evolucionários, a Lógica Fuzzy é mais uma ferramenta de inteligência computacional que atualmente está sendo adotada para auxiliar o produtor na tomada de decisão, por meio do seu uso é possível predizer variáveis respostas de interesse, como por exemplo, consumo de ração, ganho de peso, conversão alimentar, indicação de tratamento antihelmíntico, etc. Ponciano et al. (2011) salientaram que a Lógica Fuzzy é uma teoria matemática aplicada a conceitos difusos, ou seja, ao invés de utilizar números, utiliza variáveis linguísticas, palavras ou sentenças como base, as quais desempenham papel importante no tratamento da imprecisão.

Borges et al. (2017) visando automatizar o processo de análise e auxiliar o produtor na tomada de decisão, desenvolveram e implementaram um módulo computacional no Software CAPRIOVI, utilizando a Lógica Fuzzy para indicação de tratamento anti-helmíntico em pequenos 
ruminantes. Para construção do módulo, os autores definiram inicialmente as variáveis linguísticas de entrada, sendo adotadas as características ovos por grama de fezes, coloração da mucosa ocular pelo método FAMACHA $^{\odot}$ e escore de condição corporal. Posteriormente foi definida a variável linguística de saída, como sendo uma nota de 0 a 10 atribuída a cada animal analisado. De acordo com a nota atribuída, o módulo realiza uma indicação de tratamento: vermifugar, alerta e não vermifugar. Os autores asseguraram que o módulo computacional pode simular com precisão o diagnóstico humano e ser utilizado para auxiliar na indicação de tratamento e seleção de animais com maior perfil de resistência a verminose.

\section{Considerações Finais}

A adoção de ferramentas informatizadas nos sistemas de produção de pequenos ruminantes é bastante limitada. Este fato ratifica a importância de grandes investimentos em pesquisas, bem como em trabalhos que expõem a aplicação na prática dessas tecnologias nos sistemas de produção de ovinos e caprinos.

\section{Conflito de Interesse} interesse.

Os autores declaram não existir conflitos de

\section{Agradecimentos}

A Coordenação de Aperfeiçoamento de Pessoal do Nível Superior (CAPES) pela concessão da bolsa de estudos ao primeiro autor.

\section{Referências}

Barbosa, M.P.; Lopes, M.A.; Zambalde, A.L. Software para gerenciamento de rebanhos bovinos: desenvolvimento e avaliação pela Softhouse. Revista Brasileira de Agroinformática, 3(1): 13-20, 2000.

Barros, C.S.; Monteiro, A.L.G.; Poli, C.H.E.C.; Dittrichi, J.R.; Canziani, J.R.; Fernandes, M.A.M. Economic return of sheep production on pasture and in feedlot. Revista Brasileira de Zootecnia, 38(11): 2270-2279, 2009.

Borges, L.S.; Sarmento, J.L.R.; Santos Neto, P.A.; Carvalho, T.L.; Castro, O.C.C. CAPRIOVI. Programa de Computador, número de registro BR517000024-7, Instituto Nacional de Propriedade Industrial, 2017. Disponível em: <https://easii.ufpi.br/capriovi>. Acesso em: 09 jun. 2018.
Carneiro, W.P.; Ramos, J.P.F.; Pimenta Filho, E.C.; Carvalho, J.E.C.; Moura, J.F.P. Avaliação produtiva e reprodutiva de caprinos leiteiros no Semiárido paraibano. Revista Científica de Produção Animal, 18(1): 1825, 2016.

Carvalheiro, R.; Queiroz, S.A.; Kinghorn, B. Optimum contribution selection using differential evolution. Revista Brasileira de Zootecnia, 39(7): 1429-1436, 2010.

Carvalho, T.; Santos, N.; Lira, W.; Oliveira, P.A.; Santos Neto, P.; Sarmento, J. L.; Rabêlo, R. An Information System for Genetic Improvement of Goats and Sheep. Sociedade Brasileira de Computação, 1(1): 100-107, 2016.

Conrado, V.D.C.; Arandas, J.K.G.; Ribeiro, M.N. Regression models to predict the weight of Caninde goat breed through morphometric measures. Archivos de Zootecnia, 64(247): 277-280, 2015.

França, F.M.C.; Holanda Júnior, E.V.; Sousa Neto, J.M. Análise da viabilidade financeira e econômica do modelo de exploração de ovinos e caprinos no Ceará por meio do Sistema Agrossilvipastoril. Revista Econômica do Nordeste, 42(2): 287-308, 2016.

Gomes, F.M.; Pereira, F.M.; Marins, F.A.S.; Silva, M.B. Comparative study between the generalized reduced gradient and genetic algorithm in multiple response optimization. Revista Científica Eletrônica de Engenharia de Produção, 17(2): 592-619, 2017.

IBGE. Instituto Brasileiro de Geografia e Estatística. Pesquisa Pecuária Municipal. $2016 . \quad$ Disponível em: $<$ https://biblioteca.ibge.gov.br/visualizacao/p eriodicos/84/ppm_2016_v44_br.pdf >. Acesso em: 10 jul. 2018.

Jangarelli, M. Strategic mating in marker-assisted selection using multivariate analysis. Revista Ceres, 61(4): 443-450, 2014.

Kinghorn, B.P.; Shepherd, R. Mate selection by groups. Dairy Science, 81(2): 55-63, 1998.

Lôbo, R.N.B. Genecoc: programa de melhoramento genético de caprinos e ovinos de corte. Marca registrada, número de registro 826872670, Instituto Nacional de Propriedade Industrial, 2003. Disponível em: 
$<$ http://srvgen.cnpc.embrapa.br/pagina/princi pal.php>. Acesso em: 10 jul. 2018.

Martins, V.N.; Marchetti, M.E.; Garcia, R.G. Qualidade da Carne de Ovinos: depende do bem-estar do animal na produção. Cadernos de Agroecologia, 4(6): 74-81, 2015.

Mendes, C.I.C.; Oliveira, D.R.M.S.; Santos, A.R. Estudo do mercado brasileiro de software para o agronegócio. Campinas: Embrapa Informática Agropecuária, 2011. 184p.

Menezes Júnior, E.L.; Batista, A.S.M.; Landim, A.V.; Araújo Filho, J.T.; Holanda Júnior, E.V. Meat quality of sheep of different breeds of breeding terminated under two production systems. Revista Brasileira de Saúde e Produção Animal, 15(2): 517-527, 2014.

Monega, T.S.; Schmidt, A.A.; Kozakevicius, A.J. Estratégias de otimização baseadas em algoritmos genéticos com operadores migração, epidemia e relaxamento local. Brazilian Society of Computational and Applied Mathematics, 5(1): 1-2, 2017.

Nakamura, E.K.; Astolfi, G.; Ribeiro, J.M.G. Algoritmos genéticos. Estudos \& Pesquisa, 10(1): 115-123, 2005.

PECUÁRIA BRASIL ASSESSORIA. Software Pecuária Brasil Ovinos. 2015. Disponível em:

$<\mathrm{http} / / / \mathrm{www}$.pecuariabrasilassessoria.com.br/ software-ovinos.php>. Acesso em: 10 jul. 2015.

Ponciano, P.F.; Lopes, M.A.; Yanagi Junior, T.; Ferraz, G.A.S. Analysis of the environment for broiler chickens using fuzzy logic: a review. Archivos de Zootecnia, 60(1): 1-13, 2011.
Santos, N.P.S.; Sarmento, J.L.R.; Campelo, J.E.G.; Rego Neto, A.A.; Neri, V.S.; Torres, T.S. Assisted selection: proposal optimization using Genetic Algorithms. PUBVET, 6(19): 1-16, 2012.

Santos, N.P.S.; Sarmento, J.L.R.; Carvalheiro, R.; Campelo, J.E.G.; Sousa, W.H.; Figueiredo Filho, L.A.S.; Rego Neto, A.A.; Biagiotti, D. Optimum genetic contribution applied to the selection of Santa Ines sheep. Pesquisa Agropecuária Brasileira, 51(6): 745-750, 2016.

Silvestre, E.A.; Costa, M.S.; Silva, P.O.; Bajay, M.M.; Pinheiro, J.B.; Zucchi, M.I.; Campelo, J.E.G.; Britto, F.B. A note on the distribution of genetic diversity of Anglo-Nubian goats in central-northern farms of Piauí, Brazil. Revista Brasileira de Zootecnia, 44(4): 155160, 2015.

Storn, R.; Price, K. Differential Evolution: a simple and efficient heuristic for global optimization over continuous spaces. Journal of Global Optimization, 11(4): 341-359, 1997.

Teixeira, I.A.M.; Gomes, R.A.; Castagnino, D.S.; Figueiredo, F.O.M.; Härter, C.J.; Biagioli, B.; Silva, S.P.; Rivera, A.R. Technological innovation in goat production. Revista Brasileira de Saúde Produção Animal, 14(1): 104-120, 2013.

Vaisakh, K.; Srinivas, L.R. Differential Evolution Approach for Optimal Power Flow Solution. Journal of Theoretical and Applied Information Technology, 4(4): 261-268, 2008 . 